\title{
Anticipatory Engineering: anticipation in sensory-motor systems of human
}

\author{
Yoshikatsu Hayashi, Jamie Blake, and Slawomir J. Nasuto \\ Brain Embodiment Lab, School of Systems Engineering, University of Reading \\ y.hayashi@reading.ac.uk \\ http://bel.reading.ac.uk/
}

\begin{abstract}
In visual tracking experiments, distributions of the relative phase between target and tracer showed positive relative phase indicating that the tracer precedes the target position. We found a mode transition from the reactive to anticipatory mode. The proposed integrated model provides a framework to understand the anticipatory behaviour of human, focusing on the integration of visual and somatosensory information. The time delays in visual processing and somatosensory feedback are explicitly treated in the simultaneous differential equations. The anticipatory behaviour observed in the visual tracking experiments can be explained by the feedforward term of target velocity, internal dynamics, and time delay in somatosensory feedback.
\end{abstract}

Keywords: Anticipatory mode, mode transition, delayed differential equations, somatosensory feedback

\section{Introduction}

Experimental evidence concerning periodic movements suggests that in order to adapt to changing environment, humans exhibit the mode transition from the reactive to anticipatory mode as the frequency of motion increases. This behaviour is understood as one of the manifestations of the anticipatory nature of the human mind, with the purpose of overcoming the inevitable time-delays in the sensory-motor systems.

In the context of human behaviour, the previous works have reported instances where body motion may occur prior to an external reference signal in hand tracking and finger tapping tasks [?,?]. Human subjects are also able to generate motion preceding a periodic target signal [?]. These phenomena indicate that the human sensory-motor system may operate in an anticipatory mode rather than a reactive mode, when the target motion is relatively fast. Recently, extending single tapping/tracking experiments for mutual interactions, we found that the anticipatory mode leads to synchronised motion between pairs of participants [?,?].

In fact, the ability to anticipate future input seems to underpin many $\operatorname{cog}$ nitive processes. A major advantage of such anticipatory brain mechanisms is 
that they allow cognitive agents to respond adequately to fast input signals. In addition, the assumption that feedback delays are threats to successful control, being regarded as an unfortunate feature of the biological systems, seems to also favour the anticipation hypothesis. Thus, a fundamental question in cognitive neuroscience is what mechanisms might give rise to such signal processing capabilities.

The simplest visual-motor coordination laboratory experiments consist of participants being asked to minimise the positional error between a tracer and a target they are asked to track. The tracer on the display represents the hand motion by being detected by the robotic manipulator.

One of the early models of human visual-motor control was based on the well-known PID feedback control law using the relative positional error between target and tracer. The relative positional difference between target and tracer is an input signal to PID control, and hand motion is generated to minimise the relative positional error. However, this control law amounts to essentially the reactive behaviour mode. The implementation of a feedback loop does not require any modelling of the system in concern.

On the other hand, the feedforward control requires some modelling framework. For example, if the dynamics of the controlled object is known, the inverse model can be calculated to generate the controlling signal directly for desired target trajectory. However, such feedforward control violates the causality in terms of the time derivative, and can be destabilised by unknown factors in the environment.

In order to explain the anticipatory mode found in visual tracking experiments, it was shown that if the feedforward term for target velocity was included, the resulting feedback and feedforward $(\mathrm{FB} / \mathrm{FF})$ model showed precedence of tracer with respect to target motion [?]. This simple extension of the conventional feedback control successfully explained the precedence of the tracer driven by anticipatory mechanism of humans. However, although this FB/FF model can be analytically pursued to explain the anticipatory behaviour in visual tracking experiments [?], and a stability analysis identified the conditions for the stable precedence of tracer, it does not explain the synchronised motion of two subjects' dyad engaged in mutual motion interactions, i.e., when a pair of visual motor systems are coupled. This limitation is due to the fact that the FB/FF model is driven by the external dynamics and it lacks of its own internal dynamics [?].

Strong anticipation has been recently proposed as a mechanism of interaction of dynamical systems in such a way that a slave system traces the future trajectory of a master system, exhibiting the constant positive phase as if the slave were anticipating the master evolution [?]. Interestingly, in the original formulation, it is the presence of a delay in the coupling that is a necessary condition to achieve the slave's anticipation of the master's dynamics [?,?]. Note here that the slave should share identical dynamics with the master system. The positive, productive status of the delayed feedback should be emphasized in contrast to the classical feedback control law, as it may have very important implications for our understanding of how cognitive systems can predict future outcomes during 
many crucial tasks. We propose a putative mechanism enhancing the precedence of tracer with more stable conditions by incorporating the strong anticipation paradigm which employs internal dynamics and time delay in somatosensory feedback.

In this paper, we discuss the anticipatory mechanism of the control law based on the integration of visual and somatosensory information with focus on the time delay in visual processing and somatosensory feedback.

\section{Experimental method and analysis}

In order to investigate the properties of the anticipation mechanism, we performed single tracking experiments in which participants are asked to minimise the positional error between the tracer and the target. A total of 10 right handed participants performed the task. The tracer displacement on the display is proportionally related to the hand motion detected by the robotic manipulator attached to the participant.

The tracer in display was set to move along a circular trajectory of $13 \mathrm{~cm}$ diameter positioned in the plane of a display screen. The phase angle of the tracer was calculated, transmitted to the display computer, and was presented along the guideline circle on the display of the participant. The initial position of target and tracer are the same, and the target started to move along the circumference of the circle in a clockwise direction. The programmed target frequency was fixed in each trial and from trial to trial increased from $0.1 \mathrm{~Hz}$ to $2.0 \mathrm{~Hz}$ with a step of $0.1 \mathrm{~Hz}$. At each trial, the participant was asked to trace a moving target for 20 seconds with a 10 second break period. 3 trials were performed at each of the 20 frequencies.

The distribution functions of relative positional errors between target and tracer were calculated at each frequency, and were fitted by the Gaussian distributions to identify the position of the peak of the distributions.

\section{$3 \quad$ Experimental results}

Fig. ?? shows the distributions of the relative phase between target and tracer at different frequencies. The positive relative phase indicates that the tracer precedes the target position. At the frequency region of $0.2 \mathrm{~Hz}$, the peak of the Gaussian distribution is located below 0, presumably as a result of the visualmotor system time delay. However, there is a clear gradual shift towards the precedence of tracer over the target as the frequency increases above $0.4 \mathrm{~Hz}$ (Fig. ??). The same gradual shift has been observed for other 9 participants [?].

In short, we also found a mode transition from the reactive to anticipatory mode. Even though the same trend has been found in visual tracking experiments of harmonic oscillators [?], our experimental results showed that humans exhibit the transition towards anticipatory mode even though the target moves at a constant velocity along the circle guide line. This is highly significant as the simplest explanation could be that the anticipatory mode was triggered by 
the rhythmic component of external stimuli. Rather, we found that rhythmic components of hand motion were generated when the participant showed the anticipatory mode [?].

Additionally, around the frequencies around $1.6 \mathrm{~Hz}$, the distributions tend to move towards the origin as they develop large spreads indicating the subject is unable to follow the target.

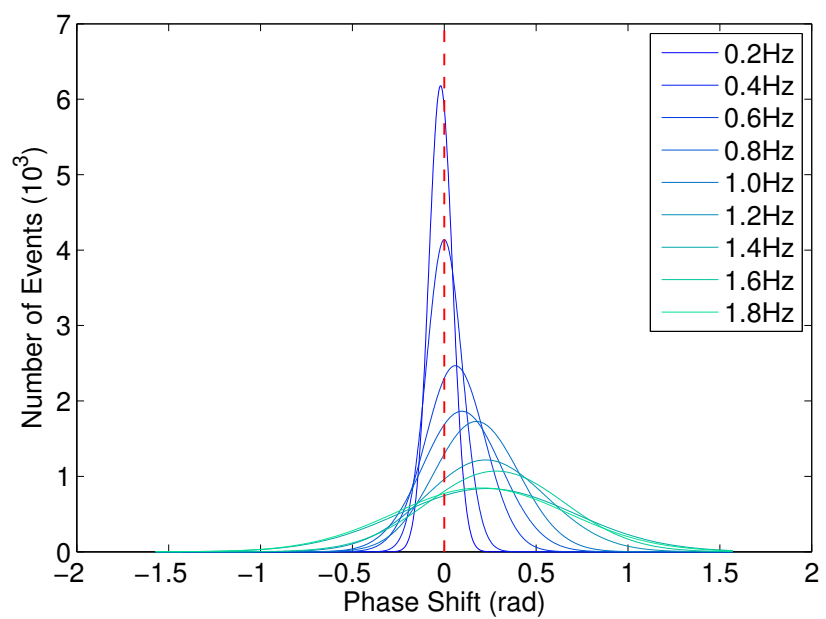

Fig. 1. Distributions of the relative phases between target and tracer across a range of frequencies for a typical subject. The positive phase indicates the precedence of tracer with respect to target.

\section{Theoretical framework for anticipatory behaviour of visual-motor systems}

In this section, we would like to discuss a theoretical framework explaining the anticipatory behaviour found in the visual tracking experiments. The aim is to provide a plausible account of a mode transition from the reactive to anticipatory mode with simple delayed differential equations, focusing on time constants and time delays in visual motor system (Fig. ??). The general signal flow in the visual motor system is assumed to comprise visual processing, motor command generation, and body dynamics. The target position $X(t)$ and the tracer position $Y(t)$ are presented on the display in front of the participant. The position of target and tracer, and the velocity of target are considered to be perceived by subjects with a time delay $\delta$ needed to register and to process the visual information.

The motor command $S(t)$ is generated by feedback and feedforward control from the visual information. A dynamics of motor command generation is given 


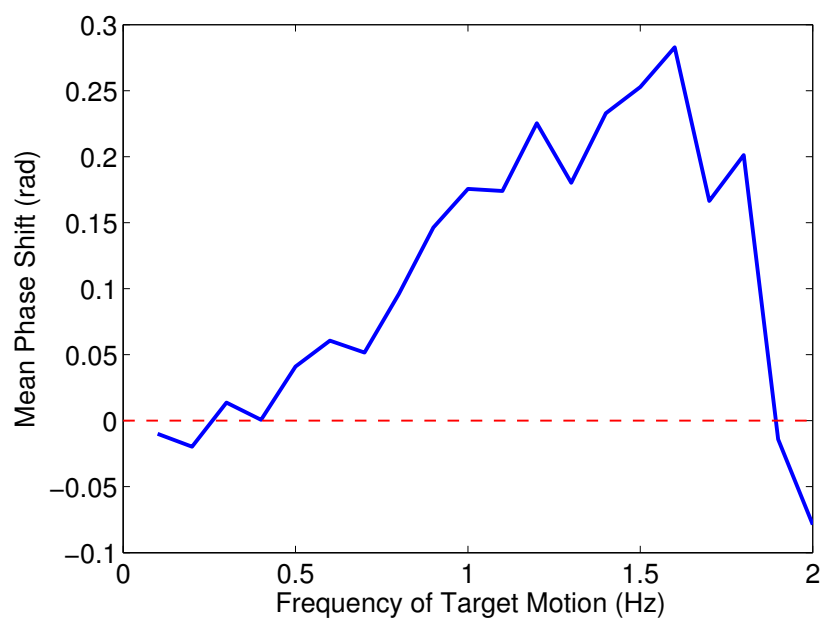

Fig. 2. Mean values of the distributions as a function of frequency for a typical subject. The positive phase indicates the precedence of tracer with respect to target.

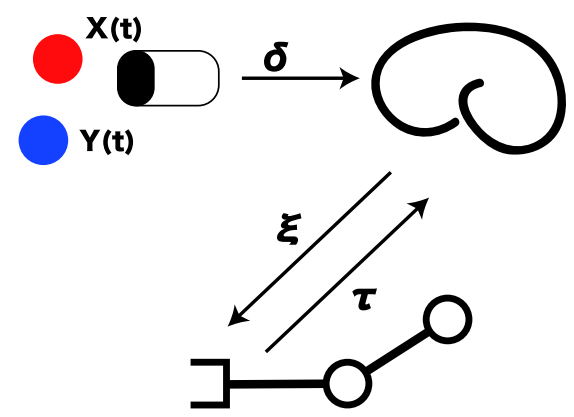

Fig. 3. Schematic picture of visual motor systems with time delays in visual and somatosensory feedback. 
by

$$
\frac{d S(t)}{d t}=\frac{1}{\tau_{v i}}[X(t-\delta)-Y(t-\delta)]+\gamma \frac{d X(t-\delta)}{d t}
$$

where $\tau_{v i}$ is a time constant of visual processing. The first term describes a feedback control for relative positional difference between target and tracer, and the second term describes a feedforward control for the target velocity with a tuning parameter $\gamma[?]$.

The signal $S(t)$ is transmitted to the hand with the time delay $\xi$, and the dynamics of hand motion $Y(t)$ is driven by a feedback control between the brain and feedback signal. Note that $S(t)$ is generated by visual information and hence is driven solely by input signals, and will fade away when visual information is no longer available.

In contrast, we assume that the dynamics of hand motion $Y(t)$ has a corresponding representation in the brain, $g(Y)$. This means that the brain has a representation of the hand dynamics which is able to generate the actual hand motion in the work space even without external inputs. This autonomous dynamics should be modulated by the brain signal representing the visual information with respect to the hand position $Y(t)$. To account for integration of sensory information, we suggest that somatosensory feedback is used to identify the hand position. This somatosensory feedback would have a time delay $\tau$ which is larger than the time delay in visual processing $(\tau>\delta)$.

Thus, the considerations above imply the hand dynamics described by

$$
\frac{d Y(t)}{d t}=g(Y)+\frac{1}{\tau_{m o}}[S(t-\xi)-Y(t-\tau)]
$$

where $\tau_{m o}$ is another time constant to process a proportional control between the brain signal and the feedback signal, and $\xi$ is the time delay to transmit the signal to hand.

In short, simultaneous equations of the visual processing and hand dynamics utilise a mechanism of adapting hand dynamics to trace target motion by integrating the visual and somatosensory feedback.

\section{Discussion}

The average relative phase between the target and the tracer, the peak location of the Gaussian distribution clearly showed the transition to the precedence of the tracer as the target frequency increased (Fig. ??). The mode transition from the reactive to anticipatory mode was found in the visual tracking experiments in which the target moves at a constant velocity.

The integrated model here provides a framework to understand the anticipatory behaviour of human movement, focusing on the integration of visual and somatosensory information. The time delays in visual processing and somatosensory feedback are explicitly treated in the simultaneous differential equations. Although, the anticipatory behaviour observed in the visual tracking experiments can be explained by the feedforward term of target velocity [?], FB/FF 
equations alone will not explain the anticipatory synchronisation between pair of participants since this model is driven by external input only.

To include the internal dynamics in hand motion, we proposed an integrated model incorporating the internal dynamics of the hand motion and the time delay in the somatosensory feedback.

Even though systematic numerical calculation of the integrated model and comparison with behavioural data is necessary to determine the constants of time delays and time constants, in the asymptotic limit of time constants of $\tau_{v i}$ and $\tau_{m o}$, the proposed model indicated a robust stability in the precedence of the tracer. Intuitively, the brain signal $S(t)$ can be considered as a virtual target in brain generated by visual processing to which a hand motion dynamics is coupled, and, the internal dynamics of hand motion represented in brain and the time delay in somatosensory feedback will further enhance the precedence of tracer with respect to the target trajectory, since the virtual target itself already shows the precedence of tracer by the feedforward term.

The stability of the FB/FF model is attributed to the Hopf bifurcation, whereas the stability of the internal dynamics model is attributed to the asymptotic relaxation to the fixed point [?]. Therefore, the proposed integrated model enhances the anticipatory horizon of precedence of own motion with respect to the target motion in visual tracking.

The model suggested here is empirical and uses the simultaneous differential equations with relevant time delays in the sensory motor system. The degrees of freedom considered in these equations are limited to a few parameters. The next question is to relate these empirical equations to brain dynamics.

From the microscopic point of view, the brain consists of billions of neurons. The neuronal networks are not static and passive systems simply responding to the external stimulation or the sensory input, but rather, spontaneous activity of individual neurons and their connectivity make it possible to generate a variety of cohesive patterns for imposed sensory-motor coupling to select a certain phase from a series of transitions of the intrinsic attractors. Consistent with this synergetics view [?], the temporal dynamics should be extracted with a few set of order parameters. Therefore, we expect that the delayed differential equations here can be validated with order parameters extracted from measurement of brain activity.

In the future study we will perform systematic numerical calculations of the integrated model to define stability conditions for the time constants and time delays leading to the stable anticipatory mode comparable with behavioural data. Also, measurement of brain activity will be performed to verify the anticipatory model proposed in this paper.

\section{References}

1. D. A. Engström, J.A. S. Kelso, and T. Holroyd, Hum. Mov. Sci., 15, 809 (1996).

2. B. H. Repp, Psychonomic Bulletin \& Review, 12-6, 969 (2005).

3. F. Ishida and Y. Sawada, Phys. Rev. Lett., 96, 168105 (2004). 
4. Y. Hayashi and Y. Sawada, Phys. Rev. E, 88 (2), 022704 (2013).

5. Y. Hayashi and T. Kondo, Phys. Rev. E, 88 (2), 022715 (2013).

6. N. Stepp and M. T. Turvey, Cogn. Syst. Res. 11-2, 148 (2010).

7. H. U. Voss, Phys. Rev. E, 61, 5115 (2000).

8. M. Ciszak, O. Calvo, C. Masoller, C. Mirasso, and R. Toral, Phys. Rev. Lett. 90, $204102(2003)$.

9. J. Blake, S. J. Nasuto, and Y. Hayashi, manuscript.

10. H. Haken, Principles of Brain Functioning: A Synergetic Approach to Brain Activity, Behavior and Cognition, Springer Publishing Company (2010).

\section{Appendix}

This work was supported by an equipment grant of University of Reading and a research grant of School of Systems Engineering, University of Reading. 